\title{
L'ESPACEMENT DES NAISSANCES ET LA MORTALITE INFANTO-JUVENILE AU RWANDA
}

\author{
Aloys ILINIGUMUGABO \\ ONAPO (Rwanda)
}

\section{INTRODUCTION}

Plusieurs études spécialisées (Boerma and al., 1984 ; De Sweemer, 1984 ; Hobcraft and al., 1983 ; Palloni and al., 1986 ; Van den Eerenbeemi, 1985) ont montré que la longueur des intervalles intergénésiques est un facteur pertinent pour l'explication des inégalités devant la mort dans la petite enfance.

En effet, l'intervalle intergénésique précédant la naissance traduit surtout les conditions de la mère pendant la grossesse dont les effets se répercutent sur l'état sanitaire du nouveau-né. L'intervalle intergénésique suivant la naissance d'un enfant affecte sa mortalité par le biais du sevrage précoce. Le raccourcissement de la durée d'allaitement au sein dans un pays où le lait maternel est la seule source de protéines disponibles pour les enfants en bas âge, allié à l'insalubrité entourant la préparation des aliments de substitution, sont à l'origine de nombreuses maladies nutritionnelles et infectieuses qui aboutissent souvent au décès des enfants.

L'espacement des naissances peut agir également sur la mortalité infanto-juvénile par le canal de la promiscuité et de la concurrence qu'il suscite entre les frères pour la nourriture, l'attention et les soins de la mère.

Dans cette étude, nous essayons de montrer que la tendance au raccourcissement des intervalles intergénésiques (Ilinigumugabo, 1989) augmente les risques de mortalité infanto-juvénile dans la société rwandaise. 


\section{PRESENTATION DES DONNEES}

Cette étude est basée sur l'analyse des données de l'enquête rwandaise sur la fécondité dont les opérations de collecte se sont déroulées entre le 22 août et le 7 décembre 1983. Cette enquête a été réalisée en totalité par l'Office National de la Population (ONAPO). Les données ont été collectées sur un échantillon probabiliste des districts stratifiés par zones éco-logiques et par préfecture selon une probabilité proportionnelle à la taille à l'intérieur des deux secteurs urbain et rural, mais avec une probabilité triple dans le secteur urbain. L'enquête rwandaise sur la fécondité était basée sur trois questionnaires: un questionnaire-ménage, un questionnaire-femme et un questionnaire-mari. Le recensement des résidents du ménage avait l'objectif principal d'identifier les femmes de 15 à 50 ans devant être soumises à l'enquête individuelle sur la fécondité. Le questionnaire-femme correspond exactement au questionnaire de base(1) de l'Enquête Mondiale sur la Fécondité (E.M.F.) avec le module sur les facteurs autres que la contraception qui affectent la fécondité (FOTCAF) auquel on a ajouté un autre module spécifique au Rwanda réservé à l'appréciation de la situation démographique du pays. Un mari sur quatre des femmes en union monogamique a subi une enquête sur la connaissance et la pratique de la contraception et sur l'appréciation de la situation démographique du Rwanda. L'ensemble des questionnaires a été traduit en kinyarwanda, la langue maternelle de tous les Rwandais. Au total, 5.739 femmes ont été enquêtées dont 1.612 célibataires sans enfants. Le tableau 1 reprend la répartition des femmes enquêtées selon l'âge, le niveau d'instruction, le degré d'alphabétisation, la nature du lieu de résidence et la préfecture de résidence.

\section{LA STRATEGIE D'ANALYSE}

L'analyse de la mortalité dans l'enfance à partir des données sur l'histoire génésique des femmes en âge de procréer pose plusieurs problèmes théoriques dûs à la fois aux biais de troncature et de sélection, et à la mauvaise datation des décès (Rutstein, 1984). Les biais de troncature sont de même nature que ceux qui affectent l'analyse des intervalles intergénésiques (Rodriguez et Hobcraft, 1980) à la différence que les enfants en intervalles ouverts sont ceux qui étaient encore en vie au moment de l'enquête.

(1) Pour une description détaillée des questionnaires, voir W.F.S. (1975), Questionnaire de base, Documentation de base $n^{\circ} 1$ et W.F.S. (1979), Les modules de l'E.M.F. : Avortement facteurs autres que la contraception affectant la fécondité - Planification familiale - Mortalité générale, Documents non-périodiques $\left.n^{\circ} 19\right)$ Pour la version en kinyarwanda, voir ONAPO (1985) 
54.

\begin{tabular}{|c|c|c|}
\hline & $\therefore$ & 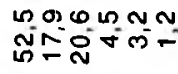 \\
\hline & $\boldsymbol{u}$ & 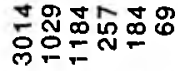 \\
\hline & ๙ீ & 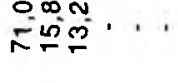 \\
\hline
\end{tabular}

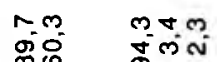

กัด

8

సิํํำ ㅎํㅇํำ

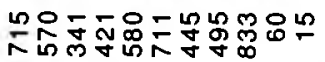

ู่ंก

कू

m

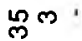

กั

$\hat{N}^{\infty}$

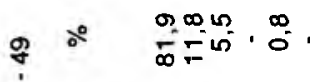

o. ㅁ on-

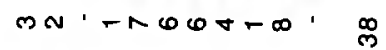

$\stackrel{\infty}{\infty}$

क्ल०

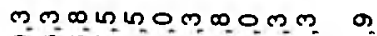

y

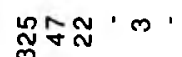

เก

กตm

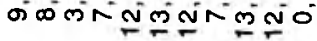

6

mஸ́,

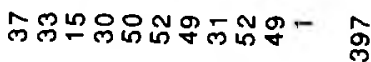

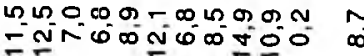

$\hat{a}^{\text {No }}$

面 \&

o

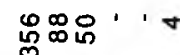

웅요

무음

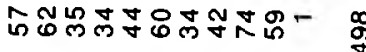

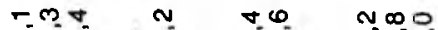

등 홈

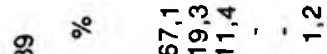

$\nabla \infty$

人

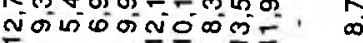

88

है

w

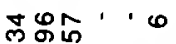

in

के

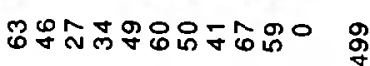

nNM $\forall 0$ DN

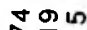

+chnmonmo

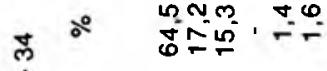

ธิ่อ

केंn

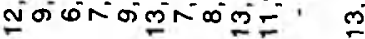

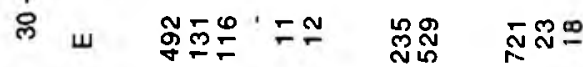

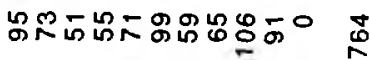

음 응

을

ก ค

$0 r=0$

m. ora

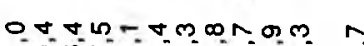

सNA

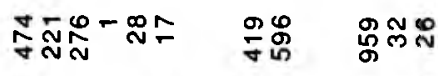

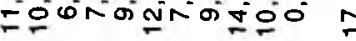

ธ

은

옹

ถั่

용

它

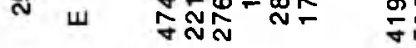

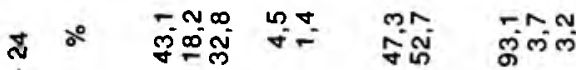

m) $-\pi N$

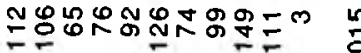

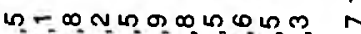

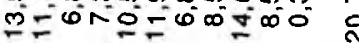

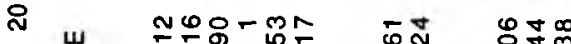

ஜ 守

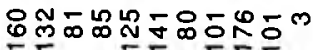

$\stackrel{\infty}{\Phi}$

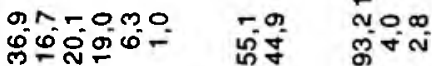

- Mognino

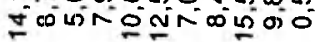

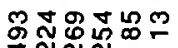

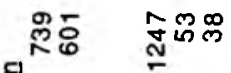

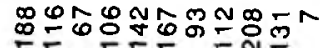

$\stackrel{\infty}{\stackrel{్}{్}}$
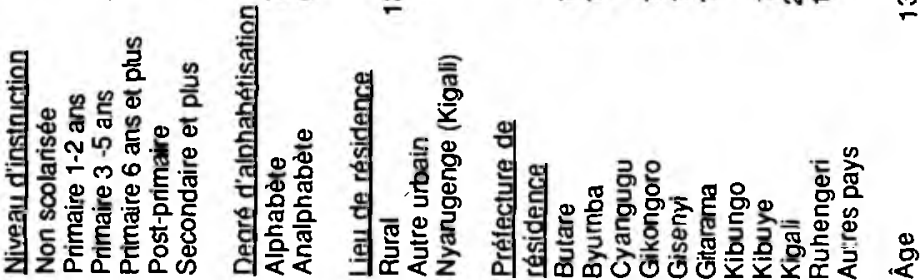
Cette source de données a la particularité d'augmenter les effets de sélection parce qu'elle ne permet d'analyser que le sort des enfants dont les mères sont encore en vie. En d'autres termes, l'utilisation de ce type de données ne tient pas compte des enfants qui sont devenus orphelins de mère. Il ne fait aucun doute que les enfants qui perdent leurs mères pendant leur jeune âge constituent un groupe à très haut risque de mortalité, surtout dans les pays du Tiers-Monde où le lait maternel est la seule nourriture disponible pour le nourrisson. Dans une analyse du niveau de mortalité, ce biais peut compromettre la généralisation à tous les enfants qui naissent dans une population donnée, notamment dans le Tiers-Monde où la mortalité maternelle reste encore très élevée.

Outre ces biais de troncature et de sélection, les àges au décès recueillis dans les histoires génésiques des femmes rwandaises enquêtées ont été très affectés par le phénomène d'attraction des multiples de six mois (tableau 2 et la figure 2). Ce phénomène signifie que la plupart des enquêtées ne connaissant pas l'âge au décès de leurs enfants ont dû faire des approximations en utilisant l'année comme unité de mesure. Pour minimiser ces effets de mémoire déficente, nous avons choisi d'analyser les proportions d'enfants survivants au moment de l'enquête parmi tous ceux qui sont nés an même moment plutôt que de baser cette analyse sur l'âge au décès déclaré. Ce choix a déterminé la méthode que nous avons utilisée pour analyser les probabilités d'échapper à la mort pendant la petite enfance. Nous avons analysé ces proportions d'enfants survivants parmi tous ceux qui sont nés au même moment par le modèle de régression logitlinéaire. Il convient de souligner que si les âges au décès déclarés étaient de bonne qualité, le modèle de régression des risques proportionnels instantanés serait préférable (Trussel et al., 1983). LINEAIRE

$\mathrm{Si} \mathrm{Pi}$ désigne la proportion d'individus appartenant à la sous-population i qui ont réalisé l'évènement étudié (le sevrage ou la reprise du cycle menstruel de la mère) que l'on suppose survenir dans cette population selon une loi logistique, on définit le logit $\lambda_{j}$ par la quantité :

$$
\lambda_{i}=\operatorname{logit}\left(P_{i}\right)=\log \left(\frac{P_{i}}{1-P_{i}}\right)
$$


56. Tableau 2 : La répartition des enfants selon leur âge au décès déclaré (enfants décédés
avant l'áge de cinq ans)

\begin{tabular}{|c|c|}
\hline Age (en mois) & Pourcentage des décès \\
\hline 0 & 28,1 \\
\hline 1 & 4,7 \\
\hline 2 & 3,3 \\
\hline 3 & 3,0 \\
\hline 4 & 2,2 \\
\hline 5 & 1,5 \\
\hline 6 & 2,8 \\
\hline 7 & 1,6 \\
\hline 8 & 1,7 \\
\hline 9 & 1,2 \\
\hline 10 & 0,4 \\
\hline 11 & 0,3 \\
\hline 12 & 6,3 \\
\hline 13 & 0,5 \\
\hline 14 & 1,4 \\
\hline 15 & 0,8 \\
\hline 16 & 0,8 \\
\hline 17 & 0,9 \\
\hline 18 & 3,6 \\
\hline 19 & 0,4 \\
\hline 20 & 0,4 \\
\hline 21 & 0,2 \\
\hline $22-23$ & 0,1 \\
\hline 24 & 8,4 \\
\hline 25 & 0,2 \\
\hline 26 & 0,2 \\
\hline 27 & 0,4 \\
\hline 28 & 0,5 \\
\hline 29 & 0,3 \\
\hline 30 & 2,0 \\
\hline 31 & 0,2 \\
\hline 32 & 0,1 \\
\hline $33-35$ & 0,1 \\
\hline 36 & 5,9 \\
\hline $37-39$ & 0,2 \\
\hline 40 & 0,2 \\
\hline 41 & 0,2 \\
\hline 42 & 0,4 \\
\hline $43-47$ & 0,1 \\
\hline 48 & 3,1 \\
\hline $49-53$ & 0,3 \\
\hline 54 et + & 1,1 \\
\hline TOTAL & 100,0 \\
\hline
\end{tabular}


57.

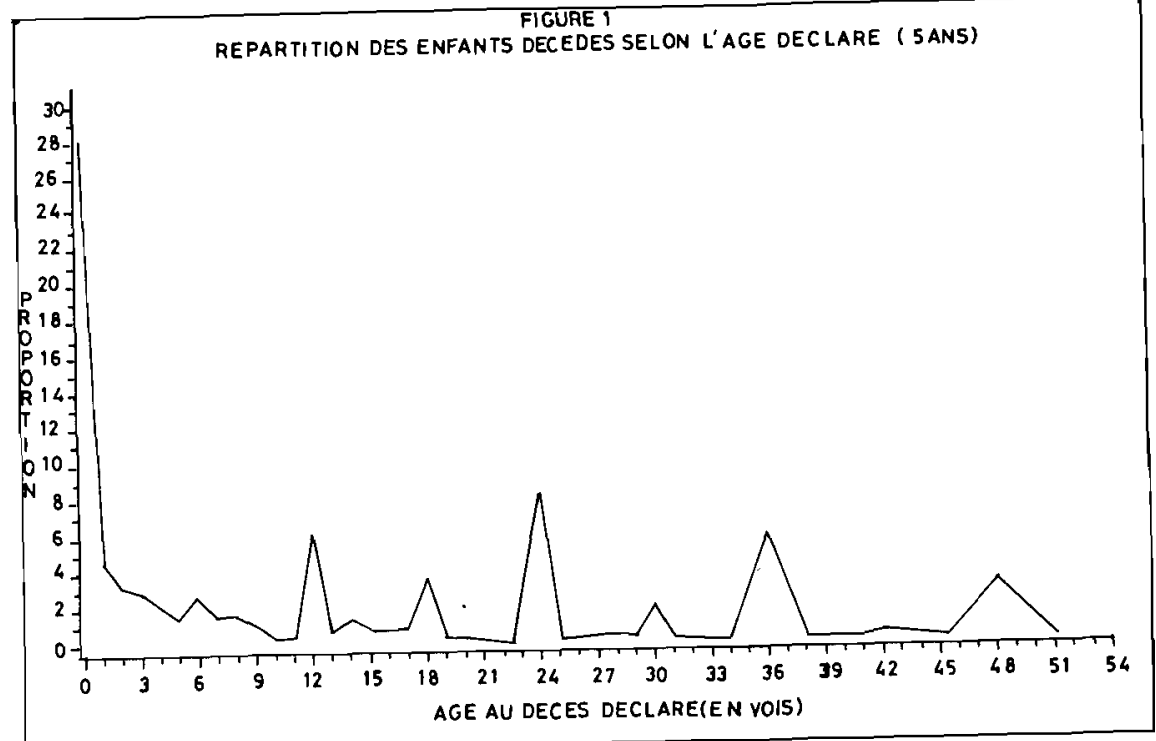


Le modèle de régression logit-linéaire est formellement du type (Fienberg, 1980) :

$$
\lambda_{i}=\beta_{0}+\sum_{j=1}^{m} \beta_{i} Z_{i j}+e_{i} \quad i=1,2, \ldots, N
$$

où est le terme indépendant, ei celui de l'erreur résiduelle et les $\beta_{j} \quad(j=1, \ldots, m)$ les paramètres logistiques à estimer par la méthode du maximum de vraisemblance. Les quantités zij sont des valeurs des $m$ variables explicatives pour le ième individu observé.

Sous cette formulation, les $m$ variables explicatives peuvent être des variables continues ou dichotomiques. Lorsqu'une variable explicative est qualitative, cette formulation suppose la création de variables artificielles binaires (dummy variables) associées à chaque modalité(2).

A partir des estimations des coefficients logistiques $\beta \mathrm{i}$, on calcule ensuite les probabilités (ou proportions) estimées pour que l'évènement étudié se réalise dans chacune des sous-populations de la matrice de contingence par la fonction anti-Logit. Les probabilités estimées sont calculées par l'expression :

$$
\hat{P}_{i}=\frac{\exp \left(\hat{\beta}_{0}+\sum_{i=1}^{m} \hat{\beta}_{i} Z_{i j}\right)}{1+\exp \left(\hat{\beta}_{0}+\sum \hat{\beta}_{j} Z_{i j}\right)}
$$

Les résultats ainsi obtenus par le modèle logit-linéaire peuvent s'interpréter à l'aide de coefficients logistiques $\left(\beta_{\mathrm{i}}\right)$ ou en termes de probabilités estimées $(\mathrm{Pi})$. Lorsque les variables explicatives sont qualitatives, la somme des paramètres logistiques associés aux variables dichotomiques artificielles correspondant aux différentes modalités d'une variable qualitative est égale à zéro. Dans un modèle logit-linéaire, les effets sont supposés multiplicatifs au niveau des risques relatifs (odds ratio) et ils deviennent additifs par la transformation logistique. Dès lors, dans la suite, nous présenterons les $(\mathrm{k}-1)$ premiers paramètres pour une variable à $\mathrm{K}$ modalités, le kième s'obtenant par la différence de la somme des autres à l'unité.

Le signe et la grandeur des paramètres logistiques associés aux différentes modalités d'une seule variable qualitative (ou catégorique) montrent le sens de la relation entre la variable considérée et le comportement étudié. L'estimation du niveau de cette relation requiert souvent le recours à un indice de mesure d'association qui permet de synthétiser les multiples probabilités estimées surtout lorsque le modèle comporte plusieurs variables explicatives. Confied (1951) a proposé une mesure d'association à partir des proportions qui fournit une approximation des risques relatifs, the odds ratio, que nous utilisons dans cette étude (Flein, 1973). Cette notion de risque

2 pour une description détaillée de la dichotomisation des variables qualitatives dans un modèle logit-linéaire, voir SAS User's Guide : Statistics. version 5 Edition, p. 192-196 
relatif exprime le risque encouru par un individu appartenant à la catégorie i par rapport à celui appartenant à la catégorie $\mathrm{j}$ vis-à-vis de l'évènement étudié.

Si deux individus qui se caractérisent respectivement par les modalités i et $\mathrm{j}$ d'une variable explicative donnée sont par ailleurs identiques par rapport aux autres variables explicatives, l'approximation du risque relatif de l'individu de la modalité i lorsque la modalité j sert de référence est approchée par la quantité $\mathrm{Ri} / \mathrm{j}$ :

$$
R_{i / j}=\frac{P_{i}}{1-P_{i}} \frac{1-P_{i}}{P_{j}}
$$

Dans un modèle logit-linéaire, cette approximation du risque relatif dans la sous-population qui se caractérise par la modalité $\mathrm{i}$ si celle définie par la modalité j sert de référence devient :

$$
\hat{R}_{i / j}=\exp \left(\hat{\beta}_{i}-\hat{\beta}_{j}\right)
$$

Dans la suite, cette dernière expression nous servira à analyser les résultats du modèle logitlinéaire parce qu'elle facilite des effets propres des variables initiales sans devoir recourir aux probabilités estimées (ou proportions estimées) dont la présentation sous forme de tahleaux est complexe lorsque le modèle comporte plus de trois va-iables. Cependant, les probabilités estimées seraient indispensables pour interpréter les effets d'intéraction.

Le coefficient de corrélation multiple qui montre la variance expliquée dans une analyse de régression classique n'est pas disponible dans un modèle de régression logit-linéaire. Dans ce dernier on recourt au rapport des vraisemblances. Pour tester si une variable explicative donnée contribue de façon significative à améliorer la portée explicative du modèle, on procède à l'analyse des rapports des vraisemblances par le test du Chi-carré $\left(\chi^{2}\right)$.Cette analyse est souvent assimilêe à l'analyse de la variance expliquée (Goodman, 1972 ; Pebley, 1981).

IV.

\section{PRESENTATION DES RESULTATS}

Après cette précision méthodologique, revenons à l'objet de cette analyse. L'indicateur de l'espacement des naissances retenu est l'intervalle intergénésique précédant la naissance de l'enfant de référence. Pour des raisons que nous avons exposées précédemment, nous analysons le sort subi par toutes les naissances vivantes survenues pendant les cinq dernières années précédant l'enquête en utilisant la variable dichotomique de scores 0 et 1 correspondant respectivement à l'enfant déjà décédé au moment de l'enquête et à l'enfant encore en vie à ce moment. Pour déterminer le modèle à analyser nous avons procédé à une étude préliminaire des tests de rapports des vraisemblances pour plusieurs caractéristiques d'identification de la mère 
60.

tels que l'âge à la naissance de l'enfant, la parité atteinte à la naissance de l'enfant, la nature du lieu de résidence, la préfecture de résidence, le niveau d'instruction, la religion, l'ethnie, l'activité professionnelle, le type de légitimité sociale de l'union conjugale dans laquelle l'enfant est né. Cinq variables se sont révélées statistiquement significatives au seuil de confiance de $5 \%$ : l'âge de l'enfant au moment de l'enquête, l'âge de la mère à la naissance de l'enfant, l'intervalle intergénésique précédant la naissance de l'enfant, le niveau d'instruction de la mère et l'activité professionnelle de la mère. La forte corrélation qui existe entre ces deux dernières variables engendre des effets de multicollinéarité lorsqu'elles sont contrôlées simultanément qui provoquent des estimations erratiques des paramètres qui leur sont associés. Nous avons retenu le niveau d'instruction qui expliquait une plus grande proportion de la variance. Le nombre de modalités pour chaque variable a été réduit pour éviter une subdivision trop exagérée de l'échantillon. Elles sont reprises dans le tableau 3.

Avant d'adopter le modèle dont nous présentons les résultats, nous avons d'abord testé la contribution à l'explication de la "deviance"(3) des différentes interactions entre les quatre variables indépendantes du modèle prises deux à deux. Le test de chi-carré a conduit à retenir les variables initiales uniquement. L'analyse de la déviance et les paramètres estimés par la modèle de régression logit-linéaire sont repris dans les tableaux 4 et 5.

Le but de cette analyse n'étant pas de quantifier le niveau de la mortalité infantile et juvénile au Rwanda, il nous parait préférable d'interpréter d'abord ces résultats en termes de risques relatifs avant de présenter les proportions estimées des enfants décédés (ou leur complément à Tunité : les proportions des enfants encore en vie). Comme nous l'avons précisé, le modèle de régression logit-linéaire ne permet pas d'estimer directement les risques relatifs. Nous avons adopté l'indice d'association proposé par Cornfield (1951), odds ratio comme une approximation des risques relatifs.

Le calcul de ces approximations des risques relatifs aboutit aux résultats repris dans le tableau 6.

Il ressort de ces indices d'association que la longueur de l'intervalle intergénésique précédant une naissance vivante est un facteur de discrimination très important face au risque de mourir dans l'enfance au Rwanda. Si on choisit le groupe d'enfants dont les mères ont réalisé un intervalle intergénésique supérieur à trois ans comme la catégoric de référence, les risques relatifs de mortalité augmentent fortement lorsque l'intervalle intergénésique se raccourcit(4). L'indice d'approximation des risques relatifs double lorsque l'intervalle intergénésique précédant la naissance est compris entre un an et demi et deux ans, il quadruple presque $(3,8)$ lorsque l'intervalle intergénésique descend en dessous d'un an.

(3) Si Lc est la vraisemblance estimée par le modèle considéré et Ls la vraisemblance estimée du modèle saturé correspondant, le rapport des vraisemblances (Deviance) est défini par la quantité

$$
\chi_{L^{2}}=-2 \log \left(L_{c} / L_{s}\right)
$$

(4)Hobcraft et al. (1983) ont trouvé des résultats similaires lors de l'analyse de quelques récentes enquêtes africaines sur la fécondité (Sénégal, Lesotho, Kenya et Ghana). 
61.

Tableau 3 : Les variables retenues dans l'analyse des proportions des enfants déjâ décédés au moment de l'enquête et leurs modalités.

\section{La variable}

La durée écoulée dépuis

la naissance de l'enfant

(son âge s'il est vivant)

L'âge de la mère à la

naissance de l'enfant

Le niveau d'instruction de la mère

L'intervalle intergénésique précédant la naissance de l'enfant de référence
Code de la variable

AGE

AMNI1

BINT

$1=$ intervalle de moins de 12 mois

$2=$ intervalle compris entre 12 et 18 mois

$3=$ intervalle compris entre 18 et 24 mois

$4=$ intervalle compris entre 24 et 30 mois

$5=$ intervalle compris entre 30 et 36 mois

$6=$ intervalle supérieur à 36 mois 
62.

Tableau 4 : Les tests de la vraisemblance expliquée (Deviance) par les différentes variables retenues dans le modèle logit-linéaire pour analyser les proportions d'enfants décédés au moment
de l'enquête

Variable

Paramètre indépendant

Durée écoulée depuis la naissance de l'enfant (AGE)

Age de la mère à la naissance de l'enfant (AMN1)

Niveau d'instruction de la mère (ETUDE)

Intervalle intergénésique précédant la naissance de l'enfant (BINT)

\section{Degré de liberté}

1

4

1

2

5

\section{Chi-carré Seuil de} confiance

$340,37 \quad 0,0001$

$92,90 \quad 0,0001$

$5,28 \quad 0,02$

$17,59 \quad 0,0002$

$97,85 \quad 0,0001$ 
63.

Tableau 5 : Les paramètres estimés par le modèle de régression logit-linéaire dans l'analyse des proportions d'enfants déjà décédés au moment de l'enquête.

Le paramètre L'estimation L'erreur standard

Le paramètre indépendant

$-2,0725$

0,1123

La durée écoulée depuis la

naissance de l'enfant (AGE)

AGE (1)

AGE (2)

$-0,7546$

0,1044

AGE (3)

$-0,2636$

0,0908

AGE (4)

0,1232

0,0872

0,4240

0,0796

L'âge de la mère à la

naissance de l'enfant AMNI(1)

$-0,1180$

0,0513

Le niveau d'instruction de

la mère (ETUDE) : ETUDE (1)

0,4451

0,1104

ETUDE (2)

0,1964

0,1139

L'intervalle intergénésique prếcédant la naissance de

l'enfant (BINT) :

BINT(1)
BINT(2)
BINT(3)
BINT(4)
$\quad$ BINT(5)

0,6874

0,1506

0,5044

0,0984

0,1396

0,0896

$-0,2464$

0,0892

- 0,4449

0,1084 
64.

Tableau 6 : L'approximation des risques relatifs à partir des résultats du modèle de régression logit-linéaire

La variable et ses modalités

1. Age de la mère à la naissance

- moins de 35 ans

- 35 ans et plus

2. Niveau d'études de la mère

- la mère n'a jamais été à l'école

- la mère a fréquenté l'école primaire uniquement

- la mère a suivi un enseignement post-primaire

3. La durée de l'intervalle intergénésique précédant la naissance

- moins de 12 mois

- 12-17 mois

- 18-23 mois

- 24-29 mois

- 30-35 mois

- 36 mois et plus
Les risques relatifs de décès

1,26

1,00 
65.

Par ailleurs, le niveau d'instruction de la mère est aussi un facteur déterminant face au risque de mourir dans l'enfance au Rwanda. Le risque relatif de mortalité des enfants nés de mères qui ont fréquenté l'école primaire serait de $20 \%$ moins élevé que celui des enfants nés des mères qui n'ont jamais été à l'école. Les effets du niveau d'instruction de la mère deviennent très importants lorsque la mère a accédé à l'enseignement post-primaire. Dans cette dernière catégorie d'enfants, le risque relatif diminue de deux tiers si on maintient la catégorie des enfants nés des mères illétrées comme la catégorie de référence.

L'intervalle intergénésique précédant la naissance de l'enfant et le niveau d'instruction de sa mère ont un impact beaucoup plus important sur son risque de mortalité dans l'enfance que l'âge de sa mère à sa naissance dans la société rwandaise. Cependant, cette dernière variable cré des différences statistiquement significatives lorsqu'on distingue les mères âgées de moins de 35 ans à l'accouchement de celles qui dépassent cet âge à la naissance de l'enfant. Si on distingue ces deux catégories de mères, les enfants qui naissent de mères âgées d'au moins 35 ans auraient un risque de mourir de $27 \%$ plus élevé que celui des enfants qui présentent les mêmes caractéristiques définies par les trois autres variables du modèle mais nés de mères âgées de moins de 35 ans.

L'analyse des probabilités estimées d'être décédé aux différents âges révolus (ou leurs compléments : les probabilités de survie) confirme les inégalités de chances devant la mort pendant l'enfance associées au raccourcissement de l'intervalle intergénésique précédant la naissance et au niveau d'instruction de la mère. Les estimations des probabilités de décès pendant les cinq premières années (en âge révolu) sont reprises dans le tableau 7 (voir figure 2). Parmi les enfants nés de mères âgées de moins de 35 ans qui ont suivi un enseignement post-primaire, ceux dont les mères avaient réalisé un intervalle intergénésique précédant leur naissance inférieur à un an auraient été exposés à un risque de mourir avant l'âge de quatre ans et demi qui est estimé à $16 \%$ alors que ceux dont les mères avaient réalisé un intervalle intergénésique supérieur à trois ans, n'auraient couru qu'un risque de mourir évalué à $5 \%$. Si l'âge de la mère à l'accouchement dépasse 35 ans parmi ces enfants nés de mères qui ont suivi l'enseignement post-primaire, la proportion estimée de ceux qui étaient déjà décédés à l'âge de quatre ans et demi augmente de $6 \%$ à $19 \%$ si l'intervalle intergénésique précédant leur naissance varie d'une durée de trois ans au moins à moins d'une année.

Le niveau d'instruction de la mère crée aussi des différences très marquées entre les probabilités estimées d'échapper à la mort pendant l'enfance au Rwanda. Si l'intervalle intergénésique précédant la naissance est inférieur à un an, la proportion des enfants dont les mères, âgées de moins de 35 ans lors de leur naissance, ont suivi un enseignement post-primaire qui étaient 
déjà morts à l'âge de quatre ans et demi est estimée à $16 \%$ pour ceux qui sont nés de mères du même âge qui n'ont jamais été à l'école. Ces proportions deviennent $5 \%$ contre $13 \%$ lorsque l'intervalle intergénésique précédant la naissance de l'enfant est supérieur à trois ans. L'analyse du tableau 7 (figure 3) montre aussi que les enfants qui naissent de mères d'âge plus avancé sont plus exposés à la mortalité précoce que ceux dont les mères sont encore jeunes.

V.

CONCLUSION

Cette analyse permet de conclure que le rythme de reproduction d'une femme rwandaise constitue un facteur très important pour la survie de ses enfants pendant leur enfance quelle que soit sa catégorie sociale. On peut admettre raisonnablement que l'intervalle intergénésique précédant une naissance est fortement lié à l'intervalle intergénésique fermé qui suit cette naissance parce que le rythme de reproduction d'une femme qui ne recourt pas à la contraception varie peu entre deux parités successives en l'absence de difficultés conjugales. Néanmoins, les deux types d'intervalles intergénésiques exercent leurs effets sur la mortalitê de l'enfant de référence par des voies différentes. L'intervalle intergénésique fermé précédant la naissance d'un enfant traduit surtout les conditions sanitaires de la mère pendant la grossesse (Jeliffe, 1966) dont les effets se répercutent sur l'état sanitaire du nouveau-né (petit poids à la naissance, malformations congénitales, prématurité, etc.). Dans une société comme celle du Rwanda, où le sevrage est conditionné par une nouvelle grossesse, l'intervalle intergénésique suivant la naissance d'un enfant affecte sa mortạlité par le biais du sevrage précoce. La réduction de la durée d'allaitement maternel dans un pays où le lait maternel est la seule source de protéines disponible pour les enfants en bas âges expose ceux-ci à la malnutrition et au manque de soins maternels. En fait, les effets de ces deux types d'intervalles intergénésiques se succèdent pour déterminer le sort de l'enfant. L'espacement de longue durée des naissances successives augmente les chances de survie des enfants et le raccourcissement des intervalles intergénésiques les exposent davantage au décès. La catégorie sociale de la famille qui accueille l'enfant, mesurée dans cette analyse par le niveau d'instruction de la mère, conditionne beaucoup les chances de survie de l'enfant. Mais le raccourcissement des intervalles intergénésiques est aussi néfaste pour les enfants des femmes illettrées que pour ceux des femmes rwandaises instruites. 
Tableau 7 : Les proportions d'enfants décédés estimées par le modèle de régression logit-linéaire

(Les naissances vivantes de rang deux et plus survenues pendant les cinq dernières années précédant l'enquête)

\section{L'âge de la mère à la naissance}

Moins de 35 ans

35 ans au moins

\begin{tabular}{|c|c|c|c|c|c|c|c|c|c|c|c|}
\hline $\begin{array}{l}\text { Áge de } \\
\text { l'entant }\end{array}$ & & $\begin{array}{l}0-11 \quad 12 \\
\text { mois }\end{array}$ & $\begin{array}{l}-232 \\
\text { mois }\end{array}$ & $\begin{array}{c}-3536 \\
\text { mois }\end{array}$ & $\begin{array}{l}4748-5 \\
\text { mois }\end{array}$ & $\begin{array}{l}5900-11 \\
\text { mois }\end{array}$ & $\begin{array}{l}12-23 \\
\text { mois }\end{array}$ & $\begin{array}{l}24-35 \\
\text { mois }\end{array}$ & $\begin{array}{l}36-47 \\
\text { mois }\end{array}$ & $\begin{array}{c}48.59 \\
\text { mois }\end{array}$ & mois \\
\hline $\begin{array}{l}\text { Niveau } \\
\text { d'instruc- } \\
\text { tion }\end{array}$ & $\begin{array}{l}\text { Interval- } \\
\text { le inter- } \\
\text { génési- } \\
\text { que }\end{array}$ & & & & & & & & & & \\
\hline La mère & $<12 m$ & 0,14 & 0,21 & 0,28 & 0,35 & 0,36 & 0,17 & 0,25 & 0,33 & 0,40 & 0,41 \\
\hline n'a jamais & $12-17 m$ & 0,12 & 0,18 & 0,25 & 0,31 & 0,32 & 0,19 & 0,22 & 0,29 & 0,36 & 0,31 \\
\hline été à & $18-23 m$ & 0,02 & 0,13 & 0,18 & 0,23 & 0,24 & 0,12 & 0,16 & 0,22 & 0,28 & 0,29 \\
\hline \multirow[t]{3}{*}{ l'école } & $24-29 m$ & 0,06 & 0,09 & 0,13 & 0,17 & 0,18 & 0,07 & 0,12 & 0.16 & 0,21 & 0,22 \\
\hline & $30-35 \mathrm{~m}$ & 0,05 & 0,08 & 0,11 & 0,15 & 0,15 & 0,06 & 0,10 & 0,14 & 0,18 & 0,18 \\
\hline & $36 \mathrm{~m}$ et + & 0,04 & 0,07 & 0,09 & 0,12 & 0,13 & 0,05 & 0,08 & 0,12 & 0,15 & 0,16 \\
\hline \multirow{2}{*}{\multicolumn{2}{|c|}{$\begin{array}{l}\text { La mère a <12 m } \\
\text { fréquenté12-17m }\end{array}$}} & 0,11 & 0,17 & 0,23 & 0,29 & 0,30 & 0,14 & 0,21 & 0,28 & 0,34 & 0,35 \\
\hline & & 0,09 & 0,15 & 0,20 & 0,26 & 0,26 & 0,12 & 0,12 & 0,24 & 0,30 & 0,31 \\
\hline l'école & $18-23 m$ & 0,07 & 0,11 & 0,15 & 0,19 & 0,20 & 0,08 & 0,13 & 0,18 & 0,23 & 0,24 \\
\hline primaire & $24-29 m$ & 0,05 & 0,07 & 0,11 & 0,14 & 0,15 & 0,05 & 0,09 & 0,13 & 0,17 & 0,12 \\
\hline unique- & $30-35 \mathrm{~m}$ & 0,04 & 0,06 & 0,09 & 0,12 & 0.12 & 0,05 & 0,03 & 0,1111 & 10,14 & 0,15 \\
\hline ment & $36 \mathrm{~m}$ et + & 0,03 & 0,05 & 0,07 & 0,10 & 0,10 & 0,04 & 0,06 & 0,09 & 0,12 & 0,13 \\
\hline La mère a & $<12 m$ & 0,05 & 0,08 & 0,12 & 0,15 & 0,16 & 0,06 & 0,10 & 0,14 & 0,18 & 0,19 \\
\hline suivi un & $12-17 m$ & 0,04 & 0,07 & 0,10 & 0,13 & 0,14 & 0,05 & 0,09 & 0,12 & 0,18 & 0,16 \\
\hline enseigne- & $-18-23 m$ & 0,03 & 0,05 & 0,07 & 0,09 & 0,10 & 0,04 & 0,06 & 0,09 & 0,11 & 0,12 \\
\hline ment post & $-24-29 m$ & 0,02 & 0,03 & 0,05 & 0,06 & 0,07 & 0,03 & 0,04 & 0,06 & 0,08 & 0,08 \\
\hline primaire & $30-35 m$ & 0,02 & 0,03 & 0,04 & 0,05 & 0,06 & 0,02 & 0.04 & 0,05 & 0,07 & 0,07 \\
\hline & $36 \mathrm{~m}$ et + & 0,014 & 0,02 & 0,03 & 0,04 & 0,05 & 0,02 & 0,03 & 0,04 & 0,05 & 0,06 \\
\hline
\end{tabular}




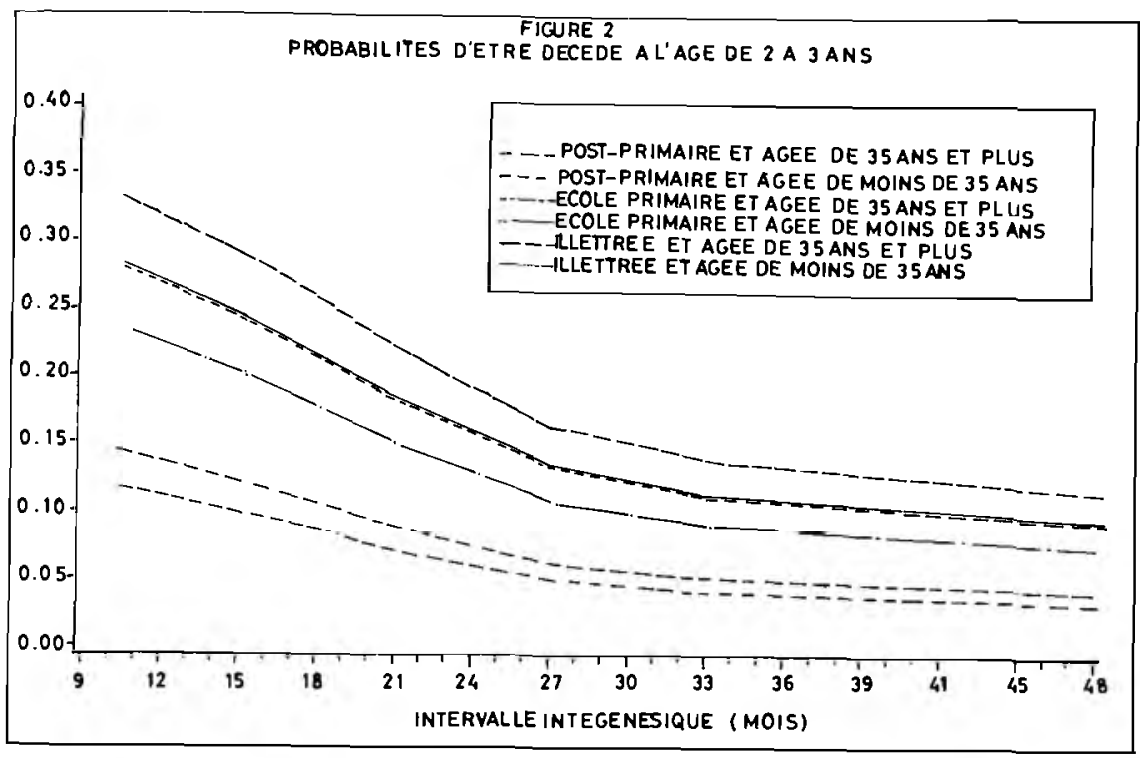


69.

\section{BIBLIOGRAPHIE DE REFERENCE}

AKOTO, E. et HILL, A.G. (1983), Morbidité, malnutrition et mortalité des enfants, in Tabutin, D. (eds), op.cit., pp. 309-334

BISHOP, Y.M., FIENBERG et HOLLAND, P.W. (1975), Discrete multivariate multivariate analysis : Theory and practice, MIT Press; Cambride, $557 \mathrm{p}$.

BOERMA, J.T. et VAN VIANEM, H.A.W. (1984), "Birth interval, mortality and growth in a rural area in Kenya", Journal of Biosocial Science. vol. 16, pp. 475-486.

CORNFIELD, J. (1951), "A method of estimating comparative rates from clinical data. Applications to cancer of the lung, breast and cervix", Journal Natl. cancer Inst.. 11, pp. 1269-1275

DE SWEEMER, C. (1984), "The influence to child-spacing on child survival", Population Studies. vol. 38(1) pp. 47- 72

FIENBERG, S.E. (1980), The analysis of cross-classified categorical data, 2nd ed., MIT Press, Cambridge-Massachussets, London, 198 p.

FLEISS, J.L. (1973), Statistical methods for rates and proportions, Wiley \& Sons, New York, 223 p.

GOODMAN, L.A. (1972), "A modified multiple regression approach to analysis of dichotomous variables", American Sociological Review, 37, pp. 28-46

HOBCRAFT, J., MCDONALD et RUTSTEIN, S.O. (1983), "Child spacing effects on infant and early child mortality", Population Index. vol. 49 (4), pp. 28-46 
HOBCRAFT, J., MCDONALD et RUTSTEIN, S.O. (1984), "Socio-economic factors in infant and child mortality : a cross-national comparison", Population Studies, vol. 38 (2), pp. 193-223

HOLFORD, T.R. (1980), "The analysis of rates and survivorship using Loglinear models", Biometrics. 36, pp. 299- 306

ILINIGUMUGABO, A. (1989), "L'espacement des naissances au Rwanda: niveaux, causes et conséquences", CIACO éditeur, Louvain-la-Neuve, $243 \mathrm{p}$.

OFFICE NATIONAL DE LA POPULATION (ONAPO), République Rwandaise (1985), "Enquête nationale sur la fécondité-

Rwanda 1983", vol. 1, Analyse des résultats, 426p.

PALLONI, A. et TIENDA, M. (1986), "The effects of breastfeeding and pace of childbearing on mortality at early ages", Demographv. vol. 23(1), pp. 31-52

PEBLEY, A.R. (1981), "The age of first birth and timing of the second in Costa Rica and Guatemala", Population Studies. 35(3), pp. 387-397

RODRIGUEZ ET HOBCRAFT (1980), "Illustrative analysis of birth intervals in Colombia", WFS, Scientific Reports, $n^{\circ} 16$

RUTSTEIN, S.O. (1984), "Infant and child mortality : levels, trends and demographic differentials", W.F.S., Comparative Studies. $n^{\circ} 43$

RUTSTEIN, S.O. (1985), "Socio-economic differentials in infant and child mortality", W.F.S., Comparative Studies $n^{\circ} 46$ 
TABUTIN, D. (eds) (1988), Population et Sociétés en Afrique au Sud du Sahara, Editions L'Harmattan, Paris, 551p.

TRUSSEL, J. et HAMMERLOUGH, C. (1983), "A hazards model analysis of covariates of infant and child mortality in Sri Lanka", Demographv. 20, pp. 1-26

VAN DEN EEERENBEEMT, M.L. (1985), "Profil demographique des Peuls du Mali Central avec référence à la mortalité infantile et juvénile", in Hill, A.G. et al.

(eds), Population, sante et nutrition au Sahel. Etudes sur le bien-être de certaines communautés de l'Afrique de l'Ouest, The London School of Hygiene and Tropical Medicine, London, pp. 83-107 